\title{
Descripción de la función sexual en mujeres con alteraciones de piso pélvico en un hospital público de Santiago
}

\author{
Claudia Flores E. ${ }^{1}$, Alejandra Araya G. PhD 1 , Javier Pizarro-Berdichevsky 2 , \\ Constanza Díaz R. ${ }^{3}$, Estefanía Quevedo C. ${ }^{3}$, Silvana González L. ${ }^{4}$ \\ 1 Enfermera-Matrona, Departamento de Salud de la Mujer, Escuela de Enfermería, Pontificia Universidad Católica de \\ Chile. ${ }^{2}$ División de Obstetricia y Ginecología, Escuela de Medicina, Pontificia Universidad Católica de Chile, Complejo \\ Asistencial Dr. Sótero del Río. ${ }^{3}$ Alumna de Enfermería, Escuela de Enfermería, Pontificia Universidad Católica de Chile. \\ 4 Matrona, Unidad de Ginecología, Complejo Asistencial Dr. Sótero del Río.
}

\section{RESUMEN}

Antecedentes: Se estima que entre $25-50 \%$ de las mujeres sufrirá de alguna alteración del piso pélvico durante su vida. La función sexual es un concepto complejo y multidimensional. Se ha planteado que a mayor sintomatología de alteraciones de piso pélvico, las mujeres presentan mayores inconvenientes en su respuesta sexual. Objetivo: Describir la función sexual de un grupo de mujeres que presentan algún tipo de trastorno de piso pélvico. Método: Estudio descriptivo a 195 mujeres con diagnóstico de alteraciones de piso pélvico utilizando un cuestionario que incluía evaluación de aspectos sociodemográficos y de la función sexual con instrumento PISQ-12. Para el análisis se utilizaron medidas de tendencia central y dispersión. Resultados: $73,7 \%$ de las mujeres refieren tener algún grado de insatisfacción con su vida sexual actual afectando principalmente el área del deseo sexual. Dentro de las limitaciones en la relación sexual reportadas se encuentran: orgasmos menos frecuentes $(70,5 \%)$, dispareunia $(89,5 \%)$, problemas de erección $(40 \%)$ y eyaculación precoz $(47,4 \%)$ en sus parejas. Conclusión: Las mujeres reportan niveles de insatisfacción con su vida sexual, disminución de su deseo sexual, dolor durante la relación sexual y disminución de la intensidad del orgasmo. Es importante incorporar en la atención de este grupo de mujeres una evaluación sistemática de la función sexual, centrándose no solo en la respuesta sexual femenina, sino que también identificando aquellas limitaciones de la mujer y de la pareja que pueden estar influyendo en su satisfacción sexual.

\section{PALABRAS CLAVES: Alteraciones de piso pélvico, piso pélvico, prolapso genital}

\section{SUMMARY}

Background: It is estimated that between $25-50 \%$ of women will suffer from a pelvic floor disorder during their lifetime. Sexual function is a complex and multidimensional concept. It has been suggested that to greater symptoms of pelvic floor disorders, women have major problems in sexual response. Objective: To describe the sexual function of a group of women with any pelvic floor disorder in a public hospital of Santiago, Chile. Method: A descriptive study of 195 women diagnosed with pelvic floor disorders using a questionnaire that included assessment of sociodemographic and sexual function instrument PISQ-12. For the analysis used measures of central tendency and dispersion. Results: $73.7 \%$ of referred women have some degree 
of dissatisfaction with their current sex life mainly affecting the area of sexual desire. Within the limitations in sexual intercourse reported are: less intense orgasms $(70.5 \%)$, dyspareunia $(89.5 \%)$, erectile dysfunction $(40 \%)$ and premature ejaculation $(47.4 \%)$ in their partners. Conclusion: Women report levels of dissatisfaction with their sex life, decreased sexual desire, pain during intercourse and decreased intensity of orgasm. It is important to incorporate in the care of these women a systematic assessment of sexual function focusing not only on the female sexual response, but also identifying those limitations of women and couples that may be influencing women's sexual satisfaction.

\section{KEY WORDS: Pelvic floor disorders, pelvic floor, pelvic organ prolapse}

\section{INTRODUCCIÓN}

Los trastornos del piso pélvico incluyen incontinencia urinaria, prolapso de los órganos pélvicos e incontinencia fecal (1). Estadísticas internacionales señalan que entre un $25 \%$ a un $50 \%$ de las mujeres en el mundo sufrirá de algún tipo de alteración del piso pélvico durante su vida y se estima que más de 200.000 mujeres anualmente se someten a una cirugía por esta causa en los EEUU $(2,3)$.

Existen múltiples factores de riesgo involucrados en el desarrollo de patologías del piso pélvico (4-6), entre ellos destacan la edad, el peso del recién nacido por parto vaginal, antecedentes de cirugías en el área (7) y aspectos sociales como el menor ingreso económico (8).

La función sexual es un concepto complejo y multidimensional que incluye aspectos físicos, psicológicos, emocionales y de la pareja (9). Evidencia internacional señala que existe una asociación entre los síntomas de alteraciones de piso pélvico y función sexual; donde a mayor sintomatología, las mujeres reportaron menor libido, disminución en la excitación, menor frecuencia de orgasmos y mayor dispareunia (10), siendo este último el síntoma más frecuente $(10,11)$.

En Chile existe una publicación que describe la función sexual de mujeres con incontinencia de orina de las Unidades de Climaterio de una clínica privada (12), desconociéndose la función sexual de las mujeres con alteraciones del piso pélvico que son atendidas en el sector público de nuestro país.

El objetivo de este estudio es describir la función sexual de un grupo de mujeres que presentan algún tipo de alteración del piso pélvico, incontinencia urinaria y/o prolapso, que son atendidas en un hospital público de Santiago, Chile.

\section{METODOLOGÍA}

Estudio descriptivo realizado en un total de 195 mujeres en la Unidad de Uroginecología del Complejo Asistencial Dr. Sótero del Río, en el período comprendido entre junio y diciembre del año 2011, que cumplieroncon los siguientes criterios de inclusión: ser mayor de 18 años, diagnóstico de alteraciones del piso pélvico (prolapso o incontinencia), y vida sexual activa en los últimos seis meses previos al enrolamiento. El criterio de exclusión fue tener intervenciones quirúrgicas por alteración del piso pélvico. Para la recolección de datos se utilizó un cuestionario autoaplicado, previo proceso de consentimiento informado, con el objetivo de determinar las características sociodemográficas y de la función sexual. Del total de la muestra, 190 mujeres aceptaron contestar los aspectos relacionados a la función sexual.

Las variables estudiadas comprenden características descriptivas de las mujeres, de la patología, y de la vida sexual. Dentro de las variables descriptivas de las mujeres se midieron edad, escolaridad, estado civil, relación de pareja, tiempo de convivencia ocupación, paridad, tipo de parto, peso mayor de recién nacido, patologías concomitantes clasificadas según CIE-10 (13), y uso de medicamentos. En relación a las variables de la patología se midieron: tipo y grado de prolapso, tipo de incontinencia, latencia de síntomas, uso de pesario. La función sexual se midió con el instrumento Prolapse Incontinence Sexual Function Questionnaire (PISQ-12) que tiene 12 ítems medidos por escala de Likert (Siempre a Nunca) distribuidos en tres dimensiones: respuesta sexual femenina (5 ítems; rango 0 a 20); limitaciones de la mujer en la relación sexual (5 ítems; rango 0 a 20); y limitaciones del hombre (2 ítems; rango 0 a 8). El rango de puntaje de este instrumento es de 0 a 48 puntos, a mayor puntaje mejor función sexual. El Alfa de Cronbach para esta muestra fue de 0,77. Esta investigación contó con la aprobación del Comité de Ética del Complejo Asistencial Dr. Sótero del Río y de la Escuela de Enfermería de la Pontificia Universidad Católica de Chile. Los datos se analizaron a través del programa SPSS 19.0 y se utilizó medidas de tendencia central, dispersión y análisis de frecuencia. 


\section{RESULTADOS}

Los datos demográficos de las mujeres en estudio se presentan en la Tabla I. Cabe destacar que la mayoría de las mujeres tienen educación media completa y $46 \%$ de ellas tiene trabajo remunerado.

Respecto de las características clínicas de las mujeres, las patologías mórbidas más frecuentesfueron: $42 \%$ del sistema circulatorio (hipertensión arterial), $15 \%$ endocrinas (diabetes e hipotiroidismo), 11\% metabólicas (hiperlipidemias), 9\% trastornos mentales (principalmente depresión), 6\% del sistema respiratorio (asma y EPOC), 5\% osteomusculares (artrosis), 4\% del sistema digestivo, 4\% otras, $2 \%$ urinarias (litiasis e insuficiencia renal), y el resto neoplasias. En cuanto a la paridad, el promedio de hijos es $3 \pm 1,3$. De un total de 276 partos, $63 \%$ de ellos fueron vaginales, $20 \%$ fórceps y $17 \%$ cesárea. El peso promedio de los recién nacidos fue de $3.724 \pm 508 \mathrm{~g}$ (rango: 2000 a $5010 \mathrm{~g}$ ).

En cuanto a las alteraciones del piso pélvico, la duración de los síntomas propios de las patologías reportadas al momento de la aplicación del cues- tionario, obtuvieron una mediana de 24 meses de latencia de los síntomas.

Del total de diagnósticos de alteración de piso pélvico, un $58 \%$ de las mujeres presentaban prolapso genital y un $42 \%$ poseía algún tipo de incontinencia; siendo las patologías más frecuentes los defectos de pared anterior (31\%) e incontinencia mixta (25\%). Se presentó además un $15 \%$ IOE, $13 \%$ defectos posteriores, $11 \%$ defectos apicales, $2 \%$ defecto completo y un $3 \%$ otros diagnósticos uroginecológicos. De acuerdo a la graduación del prolapso según el sistema Pelvic Organ Prolapse Quantification (POP-Q)(14), un 30\% se encontraba sin prolapso cuantificable, $4 \%$ en estadio I, $35 \%$ en estadio II, $27 \%$ en estadio III, $5 \%$ en estadio IV. Del total de la muestra, un 10,2\% presentaban diagnósticos ginecológicos concomitantes tales como mioma de útero $(50 \%)$, metrorragia $(20 \%)$, cáncer cérvico uterino $(10 \%)$, y el resto otras patologías.

En relación a la función sexual, el promedio total, en puntos, del instrumento PISQ-12 fue de $29 \pm$ 8,2 puntos (rango: 8-47 puntos); el detalle de cada

Tabla I

\section{CARACTERÍSTICAS DEMOGRÁFICAS DE LAS MUJERES CON ALTERACIONES DE PISO PÉLVICO (n: 195)}

\begin{tabular}{lr} 
Características & \\
Edad en años, promedio \pm DS (Rango) & $52 \pm 10$ años $(18-60)$ \\
\hline Último nivel educacional alcanzado (\%) & \\
Educación media & $55,3 \%$ \\
Estudios superiores & $12,82 \%$ \\
Educación básica & $31,31 \%$ \\
Sin estudios & $0,51 \%$ \\
Tipo de ocupación (\%) & \\
Dueña de casa & $50 \%$ \\
Trabajo remunerado & $46 \%$ \\
Cesantes & $3 \%$ \\
Estudiantes & $1 \%$ \\
Estado civil (\%) & \\
Casada & $71 \%$ \\
Soltera & $20 \%$ \\
Viuda & $6 \%$ \\
Divorciada & $3 \%$ \\
Tiene pareja (\%) & \\
Sí & $92 \%$ \\
No & $8 \%$ \\
Años de convivencia, promedio \pm DS (Rango) & $24 \pm 12$ años $(1-52)$
\end{tabular}


dimensión se encuentra en la Tabla II. La descripción por ítems se presenta en la Tabla III.

En la dimensión de respuesta sexual femenina, un $7,4 \%$ de las mujeres respondió que presentaban deseo sexual siempre. Un $29,5 \%$ de ellas refirió

\section{Tabla II}

FUNCIÓN SEXUAL SEGÚN DIMENSIÓN DEL INSTRUMENTO PISQ -12 DE MUJERES CON ALTERACIONES DE PISO PÉLVICO (n: 190)

\begin{tabular}{lcc}
\hline Dimensión & $\begin{array}{c}\text { Puntaje } \\
\text { promedio } \pm \mathrm{DE}\end{array}$ & Rango \\
\hline Respuesta sexual femenina & $12 \pm 4,1$ & $1-20$ \\
Limitaciones de la mujer en & $12 \pm 5$ & $2-20$ \\
la relación sexual & & \\
Limitaciones del hombre & $6 \pm 2,1$ & $0-8$ \\
Puntaje total & $29 \pm 8,2$ & $8-47$ \\
\hline
\end{tabular}

tener excitación siempre. En relación a la variable orgasmos, un $29,5 \%$ de ellas refirió tener orgasmos siempre, siendo estos de menor intensidad en los últimos seis meses en un $81,1 \%$. Finalmente, un $26,3 \%$ del total de las mujeres refirió estar satisfecha siempre con su vida sexual actual (Tabla III).

En relación a la dimensión de limitaciones de la mujer en la relación sexual, durante la relación sexual, un $27,4 \%$ refirió dolor nunca; el $64,7 \%$ que restringe por miedo manifestó incontinencia urinaria durante el coito en algún grado; un $68,4 \%$ contestó algún grado de miedo a la fugas de orina que restringe la actividad sexual y un $63,4 \%$ de las mujeres manifestaron restricciones en sus relaciones sexuales secundarias a su prolapso. Finalmente, un $21,1 \%$ refirió tener sentimientos negativos durante la actividad sexual con su pareja (Tabla III).

Con respecto a la dimensión de limitaciones del hombre, las mujeres reportaron que en un $40 \%$ sus parejas tenían problemas de erección y un $47,4 \%$ tenían eyaculación precoz en algún momento durante la actividad sexual (Tabla III).

\section{DISCUSIÓN}

Este es el primer estudio realizado en población chilena usuaria del sistema público que describe la función sexual en mujeres con alteraciones del piso pélvico. Nuestros hallazgos muestran que un $26,3 \%$ de las mujeres refiere estar satisfecha con su vida sexual actual siempre o casi siempre, aun cuando reportan alguna limitación en ciertas áreas de su función sexual producto de las alteraciones del piso pélvico. Estos resultados no son concordantes con lo reportado en la literatura donde incluso sobre un $60 \%$ está satisfecha $(15,16)$, así mismo resultados nacionales muestran que la satisfacción es similar (12). Esto puede verse explicado por la diferencia en los instrumentos aplicados en los diferentes estudios.

El deseo sexual, dolor durante la relación sexual y la intensidad del orgasmo fueron las dimensiones de la respuesta sexual femenina más afectadas; otro estudio en población chilena concuerda en que el deseo es una de las áreas más afectadas (12). Se ha planteado que la imagen corporal puede ser un factor que explique la disminución de deseo e intensidad del orgasmo, pues las mujeres con incontinencia urinaria refieren sentirse menos deseables, incómodas, menos femeninas y avergonzadas con sus parejas producto de los síntomas de su alteración del piso pélvico (17).

Entre los factores biológicos que podrían dar respuesta a la dispareunia, se ha planteado la edad, indicando que mujeres con alteraciones de piso pélvico con menor edad tuvieron mayor dispareunia y a mayor edad menor deseo (11). Estos datos discrepan con Novi y cols (18), quienes no encontraron diferencias en cuanto a edad, trofismo vaginal, o grado de prolapso; así mismo se ha descrito que posterior al tratamiento quirúrgico el dolor se mantiene (15), sin tener claridad en las causas.

Dentro de los ítems referidos a la dimensión de limitaciones de la mujer, la pérdida de orina durante la relación sexual reportada como "siempre o casi siempre" en más de un tercio de ellas, es concordante con lo descrito en literatura $(19,20)$. No está claro el mecanismo por el cual se produce la incontinencia de orina durante el coito, pues incluso mujeres sin incontinencia de orina lo presentan (20). En esta muestra existe una diferencia discreta entre aquellas mujeres que no establecen el miedo como una limitación para iniciar o mantener una relación sexual y aquellas que lo presentan siempre o casi siempre. Liang y cols (21), reportaron que el miedo a las fugas involuntarias de orina durante la actividad sexual era un factor presente en la mayoría de las mujeres con alteraciones del piso pélvico. No obstante, otras investigaciones han mostrado que a pesar del miedo, las mujeres no evitan la actividad sexual $(15,22)$, lo que es concordante con nuestros resultados. 
Tabla III

DESCRIPCIÓN POR ÍTEMS, DE LAS DIMENSIONES DE LA FUNCIÓN SEXUAL SEGÚN PISQ-12 EN MUJERES CON ALTERACIONES DE PISO PÉLVICO (n:190)

\begin{tabular}{|c|c|c|c|c|c|c|}
\hline Dimensión & Ítem & $\begin{array}{l}\text { Siempre } \\
\text { (\%) }\end{array}$ & $\begin{array}{l}\text { Casi } \\
\text { siempre } \\
(\%)\end{array}$ & $\begin{array}{l}\text { A } \\
\text { veces } \\
(\%)\end{array}$ & $\begin{array}{l}\text { Casi } \\
\text { nunca } \\
(\%)\end{array}$ & $\begin{array}{c}\text { Nunca } \\
(\%)\end{array}$ \\
\hline \multirow{5}{*}{$\begin{array}{l}\text { Respuesta sexual } \\
\text { femenina }\end{array}$} & $\begin{array}{l}\text { ¿Con qué frecuencia usted siente } \\
\text { deseo sexual? }\end{array}$ & 7,4 & 20,5 & 51,6 & 16,3 & 4,2 \\
\hline & $\begin{array}{l}\text { ¿Usted tiene orgasmos cuando } \\
\text { tiene relaciones sexuales con su } \\
\text { pareja? }\end{array}$ & 29,5 & 26,3 & 26,3 & 8,9 & 8,9 \\
\hline & $\begin{array}{l}\text { ¿Usted se siente excitada sexual- } \\
\text { mente cuando está teniendo re- } \\
\text { laciones sexuales con su pareja? }\end{array}$ & 29,5 & 27,9 & 25,3 & 10,5 & 6,8 \\
\hline & $\begin{array}{l}\text { ¿Qué tan intensos son los orgas- } \\
\text { mos que usted ha tenido en los } \\
\text { últimos seis meses comparado } \\
\text { con lo orgasmos que usted ha te- } \\
\text { nido antes? }\left(^{\star}\right)\end{array}$ & 18,9 & 49,5 & 26,8 & 3,2 & 1,6 \\
\hline & $\begin{array}{l}\text { ¿Está usted satisfecha con el es- } \\
\text { tilo de actividades sexuales en su } \\
\text { vida sexual actual? }\end{array}$ & 26,3 & 26,8 & 28,4 & 13,2 & 5,3 \\
\hline \multirow{5}{*}{$\begin{array}{l}\text { Limitaciones de la } \\
\text { mujer en la relación } \\
\text { sexual }\end{array}$} & $\begin{array}{l}\text { ¿Usted siente dolor mientras está } \\
\text { teniendo las relaciones sexuales? }\end{array}$ & 10,5 & 11,2 & 40,0 & 11,1 & 27,4 \\
\hline & $\begin{array}{l}\text { ¿Usted tiene incontinencia de ori- } \\
\text { na mientras está teniendo activi- } \\
\text { dad sexual? }\end{array}$ & 19,5 & 11,6 & 23,2 & 10,5 & 35,3 \\
\hline & $\begin{array}{l}\text { ¿El miedo a la incontinencia res- } \\
\text { tringe su actividad sexual? }\end{array}$ & 15,3 & 15,3 & 27,4 & 10,5 & 31,6 \\
\hline & $\begin{array}{l}\text { ¿Usted evita tener relaciones } \\
\text { sexuales por el bulto a través de } \\
\text { la vagina? }\end{array}$ & 14,2 & 18,9 & 24,2 & 6,3 & 36,3 \\
\hline & $\begin{array}{l}\text { Cuando usted está teniendo re- } \\
\text { laciones sexuales con su pare- } \\
\text { ja, ¿Usted tiene alguna reacción } \\
\text { emocional negativa como miedo, } \\
\text { asco, vergüenza o culpa? }\end{array}$ & 11,1 & 10,0 & 27,4 & 5,3 & 46,3 \\
\hline \multirow{2}{*}{$\begin{array}{l}\text { Limitaciones del } \\
\text { hombre }\end{array}$} & $\begin{array}{l}\text { ¿Su pareja tiene problemas con la } \\
\text { erección que afecte su actividad } \\
\text { sexual? }\end{array}$ & 5,3 & 6,3 & 19,5 & 8,9 & 60,0 \\
\hline & $\begin{array}{l}\text { ¿Su pareja tiene problema de } \\
\text { eyaculación precoz que afecte su } \\
\text { actividad sexual? }\end{array}$ & 7,9 & 8,9 & 21,1 & 9,5 & 52,6 \\
\hline
\end{tabular}

(*) Intensidad del orgasmo en los últimos seis meses se evalúa con escala desde mucho menos intenso a mucho más intenso.

En cuanto a la dimensión de limitaciones de la pareja, un tercio de las mujeres reportaron que sus parejas presentan problemas de erección $(40 \%)$ o eyaculación precoz $(47,4 \%)$ con alguna frecuencia. Este hallazgo es relevante de considerar dado que gran parte de la función sexual de la mujer de- pende que su pareja no presente las limitaciones físicas o psicológicas, para no restringir el nivel de satisfacción sexual que la mujer puede alcanzar. La inclusión de las limitaciones de las parejas de las mujeres con alteraciones del piso pélvico es un resultado poco reportado, por lo que este hallazgo 
constituye una contribución en esta área.

Es preocupante que la mediana de tiempo de síntomas sea de dos años con un rango entre 1 y 9 años, hasta recibir atención especializada, lo que concuerda con evidencia internacional (23-25). Dentro de las explicaciones por lo que las mujeres no consultan oportunamente se encuentra que estas consideran que es un fenómeno normal (23), considerándolo como parte "normal del envejecimiento". Junto con esto, las mujeres consideran que este es un tema tabú, del cual no hablan con otras personas o que niegan sus síntomas por vergüenza. Es importante educar a las mujeres sobre los síntomas y signos de las alteraciones del piso pélvico, de modo que puedan consultar a tiempo. Así como también, es fundamental que los profesionales pregunten directamente a las mujeres por síntomas asociados a las alteraciones del piso pélvico, de forma de abrir el diálogo en un espacio de confianza para la mujer.

Algunas de las limitaciones de este estudio son que sus resultados son solo aplicables a mujeres sexualmente activas que no han tenido resolución quirúrgica de su alteración del piso pélvico. Futuras investigaciones debieran abordar el eventual cambio de la función sexual después del tratamiento médico o quirúrgico en población chilena.

Otra limitación de este estudio es que deja fuera aquellas mujeres sin vida sexual en los últimos 6 meses previos a la recolección de datos. Un estudio realizado en EEUU, mostró que aquellas mujeres sin actividad reportaban peor función sexual que aquellas sexualmente activas (10). Es fundamental que como profesionales de la salud ahondemos en las causas por las cuales las mujeres suspenden la actividad sexual. La evidencia señala que las causas más frecuentes para suspender la actividad sexual son los aspectos físicos y emocionales producto de los cambios que la alteración del piso pélvico ha producido en ellas (15).

Por otro lado, producto de la naturaleza descriptiva de este estudio, una limitación es el hecho de no contar con la función sexual previo a presentar las alteraciones del piso pélvico, lo que impide conocer si los aspectos negativos reportados en la función sexual son explicadas por estas patologías o es una condición anterior. Es necesario generar estudios longitudinales que permitan clarificar la asociación entre función sexual y alteraciones del piso pélvico.

\section{CONCLUSIÓN}

Considerando que los aspectos de la sexualidad, de las mujeres con alteraciones del piso pélvico, es un tema que en general no se habla ni por los profesionales de la salud ni por las mujeres, es importante tenerlo en cuenta al momento de brindar el cuidado a la mujer con esta condición. Debemos proporcionar educación destinada a mejorar los aspectos deficitarios de su función sexual, poniendo énfasis en profundizar en las causas de la disminución de la intensidad de orgasmo, falta de deseo y disminución de la satisfacción sexual, incorporando no solo los componentes anatómicos, sino que también los aspectos emocionales y psicológicos que pueden estar influenciando la función sexual de la mujer con alteración del piso pélvico (25).

AGRADECIMIENTOS: La realización de este estudio fue posible gracias al financiamiento de la Dirección de Investigación de la Escuela de Enfermería (DIEE) de la Pontificia Universidad Católica de Chile, como parte del proyecto DIEE 201004.

\section{REFERENCIAS}

1. Haylen BT, de Ridder D, Freeman RM, Swift SE, Berghmans B, Lee J, et al. An International Urogynecological Association (IUGA)/International Continence Society (ICS) joint report on the terminology for female pelvic floor dysfunction. Neurourol Urodyn 2010;29:420.

2. Chughtai B, Spettel S, Kurman J, De E. Ambulatory pessary trial unmasks occult stress urinary incontinence. Obstet Gynecol Int 2012;2012:392027.

3. Chong EC, Khan AA, Anger JT. The financial burden of stress urinary incontinence among women in the United States. Curr Urol Rep 2011;12:358-62.

4. Chen GD. Pelvic floor dysfunction in aging women. Taiwan J Obstet Gynecol 2007;46:374-8.

5. Jelovsek JE, Maher C, Barber MD. Pelvic organ prolapse. Lancet 2007 24;369:1027-38.

6. Walker GJ, Gunasekera P. Pelvic organ prolapse and incontinence in developing countries: review of prevalence and risk factors. Int Urogynecol J 2011;22:12735.

7. Swift SE, Pound T, Dias JK. Case-control study of etiologic factors in the development of severe pelvic organ prolapse. Int Urogynecol J Pelvic Floor Dysfunct 2001;12:187-92.

8. Woodman PJ, Swift SE, O'Boyle AL, Valley MT, Bland DR, Kahn MA, et al. Prevalence of severe pelvic organ prolapse in relation to job description and socioeconomic status: a multicenter cross-sectional study. Int Urogynecol J Pelvic Floor Dysfunct 2006;17:340-5.

9. Jha S, Toozs-Hobson P. Prolapse and sexual function. J Assoc Charter Physiother Womens Health 2009;104:20-26.

10. Handa VL, Cundiff G, Chang HH, Helzlsouer KJ. Female sexual function and pelvic floor disorders. Obstet Gynecol 2008;111:1045-52. 
11. Handa VL, Harvey L, Cundiff GW, Siddique SA, Kjerulff $\mathrm{KH}$. Sexual function among women with urinary incontinence and pelvic organ prolapse. Am J Obstet Gynecol 2004;191:751-6.

12. Herrera Perez A, Arriagada Hernandez J, Gonzalez Espinoza C, Leppe Zamora J, Herrera Neira F. Quality of life and sexual function in postmenopausal women with urinary incontinence. Actas Urol Esp 2008;32:624-8.

13. World Health Organization. ICD-10 Interactive Self Learning Tool. Disponible en: http://apps.who.int/classifications/apps/icd/icd10training/ ICD-10\%20Death\%20 Certificate/html/index.html. Acceso: 7 junio 2012.

14. Bump RC, Mattiasson A, Bo K, Brubaker LP, DeLancey JO, Klarskov $P$, et al. The standardization of terminology of female pelvic organ prolapse and pelvic floor dysfunction. Am J Obstet Gynecol 1996;175:10-7.

15. Barber MD, Visco AG, Wyman JF, Fantl JA, Bump RC. Continence program for women research group. Sexual function in women with urinary incontinence and pelvic organ prolapse. Obstet Gynecol 2002;99:281-9.

16. Huang AJ, Subak LL, Thom DH, SK, Ragins AI, Kuppermann $M$, et al. Sexual function and aging in racially and ethnically diverse women. J Am Geriatr Soc 2009;57:1362-8.

17. Zielinski R, Low LK, Tumbarello J, Miller JM. Body image and sexuality in women with pelvic organ prolapse. Urol Nurs 2009;29:239-46.

18. Novi JM, Jeronis S, Morgan MA, Arya LA. Sexual function in women with pelvic organ prolapse compared to women without pelvic organ prolapse. J Urol 2005;173:1669-72.

19. Espuna Pons M, Puig Clota M. Coital urinary incontinence: impact on quality of life as measured by the King's Health Questionnaire. Int Urogynecol J Pelvic Floor Dysfunct 2008;19:621-5.

20. Jha S, Strelley K, Radley S. Incontinence during intercourse: myths unravelled. Int Urogynecol J 2012;23:633-7.

21. Liang CC, Lo TS, Tseng LH, Lin YH, Lin YJ, Chang $\mathrm{SD}$. Sexual function in women following transvaginal mesh procedures for the treatment of pelvic organ prolapse. Int Urogynecol J 2012;23:1455-60.

22. Liebergall-Wischnitzer M, Paltiel $O$, Hochner-Celnikier $D$, Lavy Y, Manor O. Sexual function and quality of life for women with mild-to-moderate stress urinary incontinence. J Midwifery Womens Health 2011;56:461467.

23. Azuma R, Murakami K, Iwamoto M, Tanaka M, Saita $\mathrm{N}$, Abe $\mathrm{Y}$. Prevalence and risk factors of urinary incontinence and its influence on the quality of life of Japanese women. Nurs Health Sci 2008;10:151-8.

24. Lasserre A, Pelat C, Gueroult V, Hanslik T, ChartierKastler $\mathrm{E}$, Blanchon $\mathrm{T}$, et al. Urinary incontinence in French women: prevalence, risk factors, and impact on quality of life. Eur Urol 2009;56:177-83.

25. Basson R. Women's sexual dysfunction: revised and expanded definitions. CMAJ 2005;172:1327-33. 